\title{
The stimulus suffix effect as a memory coding phenomenon
}

\author{
KATHRYN T. SPOEHR and WILLIAM J. CORIN \\ Brown University, Providence, Rhode Island 02912
}

\begin{abstract}
Two short-term memory experiments examined the nature of the stimulus suffix effect on auditory linguistic and nonlinguistic stimulus lists. In Experiment 1, where subjects recalled eight-item digit lists, it was found that a silently articulated digit suffix had the same effect on recall for the last list item as a spoken digit suffix. In Experiment 2, subjects recalled lists of sounds made by inanimate objects either by listing the names of the objects or by ordering a set of drawings of the objects. Auditory suffixes, either another object sound or the spoken name of an object, produced a suffix effect under both recall conditions, but a visually presented picture also produced a suffix effect when subjects recalled using pictures. The results were most adequately explained by a levels-of-processing memory coding hypothesis.
\end{abstract}

When subjects are asked to recall items from a list that exceeds the capacity of short-term memory, they typically show a bowed serial position function. That is, recall is better on the first few (the primacy effect) and on the last one or two (the recency effect) list items than on intermediate items. However, under auditory presentation conditions, the addition of one extra item at the end of the list impairs recall for the terminal items, even when subjects are instructed to ignore this suffix and omit it from their recall of the list. This elimination of the recency effect is known as the stimulus suffix effect (Crowder, 1967; Crowder \& Morton, 1969).

Crowder and Morton (1969; Morton, Crowder, \& Prussin, 1971) have suggested a theoretical explanation of the suffix and recency effects based on the precategorical acoustic store (PAS). PAS is a sensory storage system that may last for as long as $2 \mathrm{sec}$, and that contains auditory information in a relatively unprocessed form. Under normal list presentation conditions, the last one or two elements of the list remain in PAS at the time of recall, giving the subject an added source of information upon which to base his recall of these items. However, a stimulus suffix eliminates most, if not all, of the sensory information about the last list elements from PAS, making recall of them equal to intermediate list items whose traces have also been eliminated from PAS.

This research was supported by National Science Foundation Grant BNS76-82337 to the first author and a National Science Foundation Undergraduate Research Participation fellowship to the second author. The authors wish to acknowledge Peter D. Eimas both for providing assistance in the production of the stimuli for Experiment 2 and for giving helpful comments on many phases of this research, and Ann Lee, who collected the data for Experiment 2. Reprint requests should be addressed to Kathryn T. Spoehr, Psychology Department, Brown University, Providence, Rhode Island 02912.
The PAS explanation of the suffix effect has been supported by much empirical data. Consonant with this theory, for example, are the findings that nonlinguistic auditory suffixes fail to cause a suffix effect (Crowder, 1971), a visual suffix has no effect on an acoustically presented list (Morton \& Holloway, 1970), the magnitude of the suffix diminishes as the interval between the last list item and the suffix is increased (Crowder, 1969), and the semantic relationship between the list items and the suffix has no effect on the suffix effect (Morton et al., 1971).

An alternative explanation, offered by Massaro (1972), suggests that the recency and suffix effects result from short-term memory (STM) storage. A STM explanation attributes the recency effect to the fact that the final list items are still present in STM, making them extremely accessible for recall. The effect of a stimulus suffix is either to eliminate memory codes for these last list items from STM or to interfere with the construction of the codes for these items in some way. Salter (1975) has argued against a STM explanation on the basis of its large capacity (more than the one or two items affected by a stimulus suffix) and categorical interference effects he obtained by manipulating the categorical relationships between the suffix and the list items. However, these objections seem less severe when one considers evidence that category information may, indeed, be available in STM (Shulman, 1972), and that the stimulus suffix might have its effect on STM not by removing selected list items from storage, but by preventing a suitable STM code from ever being constructed for those items. Given the verbal nature of STM (Hintzman, 1967), the nature of that code might well be articulatory. In fact, the evidence cited above for the PAS explanation of the suffix effect is also consistent with the STM articulatory code-formation hypotheses. 
The purpose of the present two experiments is to explore the merits of both the PAS and the STM coding explanations of the suffix effect in more detail. In Experiment 1, we test these two explanations using acoustically presented linguistic stimulus lists, while we manipulate the suffix conditions according to the extent to which they would interfere either with information stored in PAS or information being coded for STM. In Experiment 2, we extend our investigation to memory for lists of acoustically presented nonlinguistic stimuli. In this experiment, we again manipulate the acoustic and STM coding properties of the suffixes.

\section{EXPERIMENT 1}

The purpose of Experiment 1 is to determine whether the recency effect is caused by the subject's ability to use additional information about final list items in PAS, or whether it is due to an increased likelihood that codes for final list items appear in STM at recall time. In order to decide, lists of eight digits were presented auditorially to subjects under one of six suffix conditions. In the no-suffix control condition, lists were presented without a stimulus suffix at the end. In the zero-card condition, a suffix was appended to the list and was presented visually by means of a card with the word "Z-E-R-O" printed out. In the 0-card condition, the suffix zero was presented visually on a card showing the single digit. In the auditory condition, the suffix was spoken aloud, but the experimenter's lips were covered by a blank card, thus affording the subject acoustic input but no direct visual input about the suffix. In the visual condition, the suffix was articulated silently, giving the subjects no acoustic suffix information but conveying visual articulatory cues. Finally, in the auditory-visual condition, the suffix was spoken aloud with the experimenter's lips in full view, giving subjects both acoustic and visual articulatory suffix cues.

The PAS and STM explanations of the suffix effect predict different patterns of suffix results for these six conditions. If the PAS explanation is correct, then only those conditions in which the suffix provides acoustic information (viz., the auditory and the auditory-visual conditions) should show a suffix effect. The four suffix conditions in which there is no acoustic information to displace the final list items from PAS should show the recency effect. The STM explanation, however, predicts that any suffix that interferes with articulatory code formation ought to produce a suffix effect. Thus, we would expect that the visual condition, in addition to the auditory and auditory-visual conditions, should show a suffix effect. The inclusion of the zero-card and 0 -card conditions provide a visual suffix baseline against which to assess the effects of the visually presented suffix with articulatory cues in the visual condition.

\section{Method}

Stimulus materials. Stimulus lists consisted of the eight one-syllable digits, $1,2,3,4,5,6,8$, and 9 , in randomly ordered sequences. Twenty lists were assigned to the no-suffix condition and were presented with no additional items. Fifteen lists were assigned to the zero-card condition, in which the suffix element, zero, was presented spelled out in .75 -in. letters on a white 4 by 6 in. card held in front of the experimenter's mouth; the 0 -card condition, in which the suffix zero was shown as a single digit printed on an index card held as in the zero-card condition; the auditory condition, in which the suffix was spoken aloud with the experimenter's lips covered by a blank index card; the visual condition, in which the experimenter silently articulated the suffix element with his lips showing; and the auditory-visual condition, in which the suffix was spoken normally (i.e., with both sound and lip movements available to subjects).

Subjects. Subjects were 20 undergraduate and graduate students at Brown University who volunteered for the experiment. Subjects were run in groups of three or four at a time.

Procedure. The experimenter was a 21-year-old male native speaker of English. Subjects were seated approximately $8 \mathrm{ft}$ from the experimenter and had full view of his head and mouth. The cards containing the lists and those used for presenting suffixes were hidden from the subject's view by a barrier. Digit lists were read by the experimenter at the rate of 2 digits $/ \mathrm{sec}$, and the suffix, in those conditions where a suffix was used, was presented as the ninth list element. Every effort was made to insure that there were no timing variations in the presentation of the suffixes across conditions. Subjects were instructed to recall the eight list elements in correct serial order in the order in which they were presented in the list and to ignore any suffix element presented. Although it was impossible to check all subjects on all trials, observation of the subjects during the recall interval indicated that they did observe the ordered report instructions.

In order to insure that subjects looked at the suffix in the three visual suffix conditions, three "catch trials" were inserted randomly in each of the five suffix conditions. On a catch trial, the suffix presented was "seven," and on those trials subjects were asked to write down the first eight letters of the alphabet rather than recall the digit list.

In the first portion of the experiment, 20 lists were presented with no suffix. In this condition, the digit lists were read aloud, followed by the experimenter's raising his finger as a signal for serial recall. The first five no-suffix lists were considered practice and were not included in the data analysis. In the second portion of the experiment, lists containing suffixes were presented in a random order; the order was changed for each group of subjects run.

\section{Results}

Subjects were able to discriminate between the suffixes zero and seven on all types of suffix trials, and no subjects made any mistakes in responding with the appropriate set of items.

Figure 1 shows the percentage of errors made in each of the six experimental conditions as a function of serial position. The six conditions appear to be highly similar for Serial Positions 1-7, with important differences at Position 8. An analysis of variance showed a reliable effect of suffix condition $[F(5,95)=19.62$, $\mathrm{MSe}=1.97, \mathrm{p}<.001]$, a reliable effect of serial position $[\mathrm{F}(7,133)=24.32, \mathrm{MSe}=2.78, \mathrm{p}<.001]$, and a significant interaction between serial position and suffix condition $[\mathrm{F}(35,655)=2.197, \mathrm{MSe}=2.42, \mathrm{p}<.001]$. Wilcoxon tests performed at the various serial 


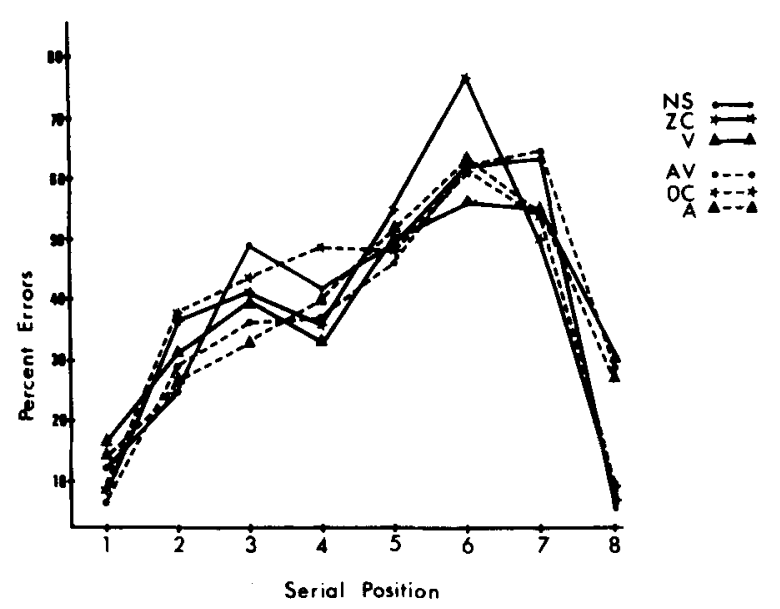

Figure 1. Percent errors as a function of serial position in Experiment 1 for the no-suffix (NS), zero-card (ZC), visual (V), auditory-visual (AV), O-card (OC), and auditory (A) suffix conditions.

positions revealed that the zero-card condition was significantly higher than the others $(p<.05)$ at Position 6 , and that the only other significant differences occurred at Position 8 , where the auditory, visual, and auditoryvisual conditions showed significantly more errors than the 0 -card, zero-card, and no-suffix conditions $(\mathrm{p}<.01)$. There were no other differences between the conditions at this position.

\section{Discussion}

It is difficult to reconcile the results of Experiment 1 with the notion that the recency effect is caused by the subject's ability to use information stored in PAS, and that the effect of a stimulus suffix is to eliminate that extra source of information for the last items. If this were the case, the lip-spoken suffix in the visual condition should not have interfered with the auditory information in PAS, and no suffix effect should have been found. The clearly interfering effect of the lipspoken suffix indicates that it must have occurred at a processing level at which the special properties of the suffix could have affected an acoustically presented list. Since neither the zero-card nor the 0 -card condition produced a suffix effect, the effectiveness of lip-spoken suffixes was clearly not due to their occurrence in the visual modality alone. Rather, the speech-like characteristics of this type of suffix appear to have caused the effect.

Although the STM coding might be the locus of the interfering effect of the articulatory cues in the visual condition, there are two other possibilities. First, as Crowder (1971) has suggested, subjects might use a subvocal "dress rehearsal" of list items between list presentation and recall. The dress rehearsal would be articulatory in nature, and it might, therefore, be expected that the presentation of any suffix with articulatory cues would prevent readout from PAS to the dress-rehearsal process. The crux of the readout explanation, however, is that PAS information is used to aid and verify the readout of the last few items. While it is true that the visual condition suffixes in the present experiment might have interfered with readout immediately upon presentation, the effect should have disappeared after the lip-spoken stimulus had been completely articulated, leaving information in PAS intact. Since the lip-spoken suffixes were completed within $1 \mathrm{sec}$ of the final list item, and the estimated duration of information in PAS is $1.5 \mathrm{sec}$ or more, subjects should have shown a recency effect in the visual condition if they were using residual PAS information to aid directed readout. At the very least, they might have shown a partial recency effect, depending upon the amount of information left in PAS after the lipspoken suffix. However, there is no indication in the data that performance at Position 8 is any better in the visual condition than in either of the two auditory suffix conditions. In fact, the visual condition is slightly worse.

A second locus of the lip-spoken interference effect might be secondary, or long-term, memory. In a series of studies on the suffix effect, Salter and his associates (Salter, 1975; Salter \& Colley, 1977; Salter, Springer, \& Bolton, 1976) have found that the magnitude of the suffix effect depends on categorical and semantic relationships between the list items and the suffix. Salter (1975) and Salter and Colley (1976) conclude that final list items that are meaningful and are not interfered with semantically by the suffix can be retrieved from a secondary memory store more easily than other list items. In the present experiment, the interference effect of the lip-spoken suffixes might be construed as a secondary, or semantic, memory interference, since it was necessary for subjects to process the suffixes thoroughly enough to decide whether they were zeroes or sevens. However, the secondary memory explanation loses credibility when one realizes that suffixes in all the conditions had to be processed to an equal semantic level. Thus, this explanation does not explain the differential suffix effects obtained in the present experiment.

The most likely cause of the interference effect, therefore, seems to be the STM coding process. As we argued earlier, material presented both acoustically and visually could be stored in primary memory in an articulatory code. Items from the end of a list that had not been coded for STM storage would be adversely affected by any type of stimulus suffix that contained articulatory cues. Thus, the results of Experiment 1 are explained.

The present data and our interpretation of it do raise questions regarding the response prefix effect, in which an extraneous item pronounced by the subject just prior to list recall increases the number of errors across all list positions but does not differentially affect the final positions (Crowder \& Morton, 1969). Since the interference of the silent articulatory gestures of the experimenter in the present visual condition must certainly have been less potent than a subject's own 
articulatory gestures made in generating a response prefix, it is not clear why our visual suffix should have had a larger influence on the end of the list than a response prefix. Following Crowder and Morton (1969), we agree that "the Stimulus Suffix Effect and the Response Prefix Effect, similar though they may superficially be, depend on different theoretical mechanisms" (p. 369). Since the response prefix affects all list positions equally, a likely possibility is that it disrupts maintenance or retrieval of all items already encoded; a stimulus suffix disrupts the encoding process itself. The articulatory apsects of a response prefix could be prevented from affecting the encoding of the final list elements if the subject is careful to pronounce the prefix only after he has finished encoding the list, or possibly, if the list elements are encoded in a nonarticulatory fashion. The present data clearly shed no light on the former hypothesis, although the issue of nonarticulatory codes is addressed in Experiment 2.

\section{EXPERIMENT 2}

Given that a stimulus suffix appears to be related to STM coding, we now demonstrate a STM interference effect for nonlinguistic auditory stimuli. In this experiment, list items consist of brief segments of commonly occurring, well known natural sounds that subjects are asked to recall under different suffix conditions. Since recalling these items requires that the subject recode the input into a more easily reportable format, it was decided to use two different types of report conditions, in order to contrast different types of recoding. In the name group, subjects were asked to report list items by writing down the names of the objects that made the sounds in the correct order. In the drawing group, subjects responded by ordering line drawings of the eight objects they had heard. It was expected that stimulus suffix effects might be different, depending upon whether subjects had to form verbal labels for the stimuli or not.

Subjects in each group recalled lists under each of five suffix conditions. In the no-suffix condition, lists were presented without a suffix item. In the sound condition, the suffix presented was another natural sound. In the name-auditory condition, the name of a sound-producing object was spoken as the ninth list item, whereas in the name-card condition, the name of a sound-producing object was written on a card and presented visually as the ninth list item. Finally, in the picture condition, subjects were shown a drawing of a sound-producing object as the ninth list item.

The PAS explanation of the suffix effect makes the same predictions about which suffix conditions should show the suffix effect for both recall groups. Only those conditions in which auditory suffix information is present (viz., the sound and name-auditory conditions) should show a suffix effect in the last list position. It is likely that the sound condition might show a stronger suffix than the name-auditory condition, given the findings of Morton et al. (1971) that the magnitude of the suffix effect depends upon the acoustic similarity between the list items and the suffix. The natural sound list items might be expected to share more acoustic properties with another natural sound than with a linguistic suffix.

If the suffix effect results from interference in STM coding, however, the extent to which the different types of suffixes in the present experiment are effective will depend on the type of recall required of the subjects. Thus, subjects in the name group would have to form a verbal code containing linguistic and perhaps articulatory information, while subjects in the drawing group would have to form a visual code that would allow them to order the pictures. A sound or an acoustically presented name suffix could interfere with the generation of either type of STM code if it affected the sensory representation of the input being used for recoding. Thus, it could produce a suffix effect for both report groups. A name-card suffix might also interfere in both recall conditions, but for different reasons. For the drawing subjects, the name-card suffix requires visual processing that might interfere with the visual code being generated for the last items of the list. For the name group, the name-card suffix could produce verbal information that could also interfere, this time with the name code, the last list item. However, the picture condition would have a different effect depending upon type of recall required. A picture suffix would not interfere with the name codes being generated in the name group and should therefore not produce a suffix effect for this group. On the other hand, a picture suffix would interfere with the STM representation of subjects in the drawing group and produce a suffix effect. Experiment 2 tests these predictions.

\section{Method}

Subjects. Subjects were 16 Brown University undergraduates, half of whom were assigned randomly to the drawing group and half to the name group. None of the subjects had participated in Experiment 1, and none had any reported hearing deficit. Subjects were paid $\$ 4$ for participating in a single $1.5 \cdot \mathrm{h}$ experimental session. All subjects were tested individually.

Stimuli and Apparatus. Ten natural sound stimuli were recorded for the experiment. Eight list stimuli included a car engine starting, a door squeaking, a typewriter, a toilet flushing, a telephone ringing, church bells chiming, a hammer pounding, and scissors cutting paper. The sounds of a stapler and of a dish breaking were also recorded to be used as suffixes. Segments of each sound of approximately 400 -msec duration were selected for presentation. Each trial of the experiment consisted of a randomly ordered sequence of the eight sound segments, separated by $500 \mathrm{msec}$ of silence. The editing and randomization was performed on the Haskins Laboratory computer. On trials where the suffix was to be a sound, the suffix item was recorded as the ninth list item on the tape. On trials where the suffix was to be a visual or spoken name, the suffix was supplied by the experimenter $.5 \mathrm{sec}$ after the last list element.

Lists were assigned to one of five suffix conditions. In the 
no-suffix condition, no extra item was presented following the list. In the sound condition, the suffix was a $400-\mathrm{msec}$ segment of a stapler. In the picture condition, the ninth item was supplied by the experimenter in the form of a line drawing of the same object that produced the sound used as the suffix in the sound condition. In the name-card condition, the name of the suffix item was spelled out on a visually presented card and in the name-auditory condition, the experimenter said the name of the suffix object approximately $500 \mathrm{msec}$ after the last list item had been heard. Stimuli were played on a Sony Model TC-377 tape recorder.

Procedure. Subjects were first familiarized with the list of possible object sounds used in the experiment: name group subjects by seeing a list of the object names, and drawing group subjects by viewing the pictures of all of the objects. Subjects then heard the list and suffix sounds one at a time, viewing the corresponding drawing or name after attempting to identify it. Subjects cycled through the list until they were able to identify each of the sounds on two successive passes through the set. All subjects were then run in the no-suffix condition, where they received 25 trials, the first 10 of which were considered practice and discarded from the data analysis. Following the no-suffix trials, subjects received 15 randomly intermixed trials in each of the sound, name-card, name-auditory, and picture conditions.

Following the list presentation on each trial in all conditions, subjects were required to recall the list items in the proper order. The name group subjects wrote down the names of the soundproducing objects; subjects in the drawing group ordered pictures of the eight objects on a rack in front of them, while the experimenter recorded the ordering from the back. The cards containing the drawings were collected and shuffled after each trial. All subjects were instructed to ignore the suffix in recalling the list items, and if the suffix was the sound of, name of, or picture of a dish rather than a stapler, they were to respond with the first eight letters of the alphabet (name group) or to turn the response cards over and order them serially by the numbers 1-8 written on the backs of the cards (drawing group).

\section{Results}

All subjects were able to discriminate successfully between the suffixes and catch suffixes, and thus no mistakes were made in responding appropriately on the catch trials.

Figures $2 \mathrm{~A}$ and $2 \mathrm{~B}$ show percent recall errors plotted as a function of serial position for each of the suffix conditions for the name and drawing groups, respectively. An analysis of variance performed on the scores for the name group showed no main effect of suffix condition $[\mathrm{F}(4,28)=1.95, \mathrm{MSe}=10.27, \mathrm{p}>.10]$, a main effect of serial position $[F(7,49)=38.65, \mathrm{MSe}=8.75$, $\mathrm{p}<.001]$, and a significan $\mathrm{t}$ interaction of serial position and condition $[\mathrm{F}(28,196)=2.67, \mathrm{MSe}=2.71, \mathrm{p}<.001]$. A Newman-Keuls test showed that for Positions 1-7, the only significant difference between suffix conditions was at Position 6 , where the sound condition was significantly greater than the picture condition $(p<.05)$. A similar test at Serial Position 8 revealed that the nosuffix condition showed significantly fewer errors than the sound, name-card, or name-auditory conditions, but was not significantly different from the picture condition $(p<.01)$. Errors in the picture condition were, in turn, significantly fewer than in the name-card and sound conditions $(p<.01)$ and were marginally fewer
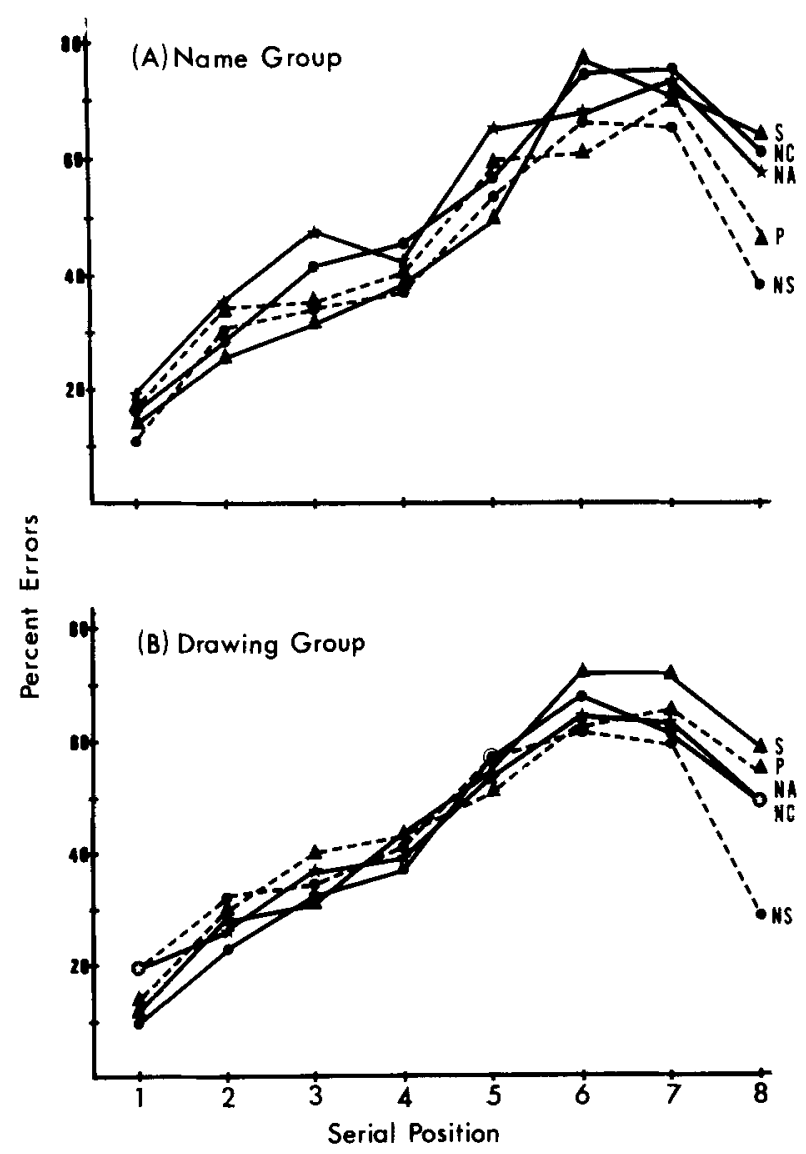

Figure 2. Percent errors as a function of serial position (Experiment 2) for (A) the name group and (B) the drawing group in the sound (S), picture (P), name-auditory (NA), namecard (NC), and no-suffix (NS) conditions.

than in the name-auditory condition $(p=.05)$. There were no other significant differences between conditions at this position.

A similar analysis performed on the results of the drawing group showed a slightly different pattem. An analysis of variance again showed no significant effect of condition $[\mathrm{F}(4,28)<1, \mathrm{MSe}=10.27]$, a main effect of serial position $[\mathrm{F}(7,49)=39.53, \mathrm{MSe}=7.32$, $\mathrm{p}<.001$ ] , and a significant interaction between serial position and condition $[\mathrm{F}(28,196)=2.84, \mathrm{MSe}=2.85$, $\mathrm{p}<.001]$. Newman-Keuls analyses showed that there were no significant differences between conditions at any of Positions 1-7, and that at Position 8, the nosuffix condition was significantly better than any of the other four conditions $(p<.01)$. There were no differences between the four suffixed conditions at Position 8.

A combined data analysis for the two groups showed that, although the drawing group made fewer errors than the name group on the average (6.61 vs. 7.08 per position in each condition), this difference was not statistically reliable $(\mathrm{F}<1, \mathrm{MSe}=210.29)$. The combined analysis showed significant effects of position $[F(7,98)=77.86, \mathrm{MSe}=8.04, \mathrm{p}<.001]$ and Position 
by Condition interaction $[\mathrm{F}(28,392)=2.67, \mathrm{MSe}=2.78$, $\mathrm{p}<.001]$, and no main effect of condition $[\mathrm{F}(4,56)=$ $1.53, \mathrm{MSe}=9.06, \mathrm{p}>.25]$. There were no significant interactions between response group and any of these factors (Fs $<1$, in all cases).

\section{Discussion}

The results of Experiment 2 confirm the findings of Experiment 1 , in that the obtained suffix effects could not have been caused by suffixes' interfering only with information held in PAS. For both groups of subjects, a visually presented suffix was just as effective as an acoustically presented suffix, as long as it interfered with the type of memory code subjects were constructing in order to carry out the recall task. Thus, for the name group, the suffixes that interfered either with identification of the last list item (sound condition) or with the construction of a verbal name for the stimuli (name-card and name-auditory conditions) yielded a suffix effect, while the other conditions did not. For the drawing group, the picture suffixes also yielded a suffix effect, since subjects in that condition also constructed pictorial codes for recall in addition to identifying the presented stimuli.

\section{GENERAL DISCUSSION}

The role of acoustic information in producing the suffix effect in the present two experiments provides a basis for a more general explanation of why different types of suffixes are effective in different situations. First, it is clear that acoustic information does play a role in producing the effect, since the auditory-visual and auditory suffix conditions were effective in Experiment 1 and the sound and name-auditory suffixes were effective in Experiment 2. However, it is equally obvious that the suffix effect is not totally dependent upon the presence or absence of acoustic information in a PAStype store, since both lip-spoken (Experiment 1) and picture (Experiment 2, drawing group) suffixes can produce the effect. Also, if suffixes acted upon codes that had been fully formed and stored in STM, we should have observed no effect of the sound or nameauditory suffixes for the drawing group in Experiment 2. It therefore must be the case that the stimulus suffix has its effect on the transfer or recoding of acoustic input into a more permanent, reportable form. The importance of the acoustic input information in this process is clear: Such recoding cannot take place if such information is absent. Thus, any suffix that degrades or eliminates the raw material of the recoding process will cause the suffix effect. As we have seen, all of the acoustic suffixes in the present two experiments caused such an effect. Moreover, the presence of auditory information must surely be responsible for the fact that recall of auditorily presented lists is superior to that of visually presented lists in the last few serial positions (Watkins, Watkins, \& Crowder, 1974), and for the fact that reading aloud a visually presented list has the same effect (Murray, 1966).

The effectiveness of nonacoustic suffixes will depend upon the level to which the subject processes the stimulus list in order to recall it (Craik \& Lockhart, 1972). In Experiment 1, subjects appear to have coded the list using verbal name labels, and thus they displayed a suffix effect with the lip-spoken suffix that interfered with that type of code. It seems likely that subjects in many other suffix-effect experiments (e.g., Crowder \& Morton, 1969; Morton et al., 1971) also made use of verbal name codes. Although it is not possible to decide with complete certainty whether the effective acoustic suffixes in these experiments interfered because of their effects on the acoustic information or because they interfered with the construction of the name code for the items, the evidence is at least consistent with the coding hypothesis. The fact that nonverbal acoustic suffixes were not effective also provides some evidence that the interference was at the level of recoding and not just an acoustic masking effect.

Processing to the level of a visual representation in order to respond pictorially opens up the possibility that a suffix can be effective at this level. Thus a visual suffix, in this case the picture suffixes for the drawing group in Experiment 2, can provide interference at this level, even though visual suffixes do not cause interference for coding at other levels (Morton \& Holloway, 1970). Salter's experiments (Salter, 1975; Salter \& Colley, 1977) provide evidence of suffix interference at the semantic level. Salter and Colley (1977), for example, have shown that if the suffix is a synonym of the final list item, the suffix effect can be reduced. Presumably, when subjects process input to the semantic level, the appropriate suffix can aid the generation of the coding of the final list item such that no interference takes place.

In summary, we have provided evidence that the interfering effect of a stimulus suffix is not limited to its interaction with acoustic information in temporary sensory storage. Rather, the suffix effect must be evaluated within the framework of the response demands made upon the subject and therefore the level at which information is being recoded and processed in the experiment.

\section{REFERENCES}

CRAIK, F. I. M., \& Lockhart, R. S. Levels of processing: A framework for memory research. Journal of Verbal Learning and Verbal Behavior, 1972, 11, 671-684.

Crowder, R. G. Prefix effects in immediate memory. Canadian Journal of Psychology, 1967, 21, 450-461.

Crowder, R. G. Improved recall for digits with delayed recall cues. Journal of Experimental Psychology, 1969, 82, 258-262.

Crowder, R. G. Waiting for the stimulus suffix: Decay, delay, rhythm, and readout in immediate memory. Quarterly Journal of Experimental Psychology, 1971, 23, 324-340.

Crowder, R. G., \& Morton, J. Precategorical acoustic storage (PAS). Perception \& Psychophysics, 1969, 5, 365-373. 
Hintzman, D. L. Articulatory coding in short-term memory. Journal of Verbal Learning and Verbal Behavior, 1967, 6. 312-316.

Massaro, D. W. Perceptual images, processing time, and perceptual units in auditory perception. Psychological Review, 1972, 79, 124-145.

Morton, J., Crowder, R. G., \& Prussin, H. A. Experiments with the suffix effect. Joumal of Experimental Psychology, 1971, 91, 169-190. (Monograph)

Morton, J., \& Holloway, C. M. Absence of a cross-modal "suffix effect" in short-term memory. Quarterly Journal of Experimental Psychology, 1970, 22, 167-176.

MURRAY, D. J. Vocalization-at-presentation and immediate recall with varying recall methods. Quarterly Journal of Experimental Psychology, 1966, 18, 9-18.

SALter, D. Maintaining recency despite a stimulus suffix. Quarterly Journal of Experimental Psychology, 1975, 27, 433-443.
Salter, D., \& Colley, J. G. The stimulus suffix: A paradoxical effect. Memory \& Cognition, 1977, 5, 257.262.

Salter, D., Springer, G., \& Bolton, L. Semantic coding versus the stimulus suffix. British Journal of Psychology, $1976,67,339-351$.

Shulman, H. G. Semantic confusions in short-term memory. Journal of Verbal Learning and Verbal Behavior, 1972, 11, 221-227.

Watkins, M. J., Watkins, O. C., \& CRowder, R. C. The modality effect in free and serial recall as a function of phonological similarity. Journal of Verbal Learning and Verbal Behavior, 1974, 13, 430-447.

(Received for publication August 4, 1978; revision accepted November 2, 1978.) 